\title{
Long non-coding RNA 00152 functions as a competing endogenous RNA to regulate NRP1 expression by sponging with miRNA-206 in colorectal cancer
}

\author{
ZHUAN-PENG CHEN ${ }^{*}$, JIAN-CHANG WEI ${ }^{*}$, QIANG WANG*, PING YANG, WANG-LIN LI, \\ FENG HE, HUA-CUI CHEN, HE HU, JUN-BIN ZHONG and JIE CAO
}

\begin{abstract}
Department of Gastrointestinal Surgery, Guangzhou Digestive Disease Center, Guangzhou First People's Hospital, Guangzhou Medical University, Guangzhou Guangdong 510180, P.R. China
\end{abstract}

Received November 8, 2017; Accepted March 21, 2018

DOI: 10.3892/ijo.2018.4451

\begin{abstract}
Colorectal cancer (CRC) is the third most common type of cancer; however, the molecular mechanisms underlying colorectal tumor metastasis and growth remain elusive. Recently, accumulating evidence has indicated that long non-coding RNAs (IncRNAs) play a critical role in CRC progression and metastasis; however, the biological role and clinical significance of IncRNA 00152 (lnc00152) in CRC remains largely unknown. Thus, in this study, Inc00152 expression was measured in 80 human CRC tissue samples, 40 non-cancerous tissue samples, and 3 CRC cell lines (SW480, SW620 and LoVo) using RT-qPCR. We examined the effects of lnc 00152 on CRC cells following transfection with lnc00152 overexpression plasmid or respective siRNA in vitro and in vivo. Luciferase assays revealed the mechanism driving competitive endogenous RNA (ceRNA). We identified that $1 n c 00152$ was aberrantly overexpressed in colorectal tumors and cancer cells and that $\operatorname{lnc} 00152$ was modulated by miRNA-206. Inc00152 overexpression enhanced the proliferative and invasive ability of CRC cells in vitro, promoted tumor growth in vivo, and was associated with the shorter overall survival of patients with CRC. In addition, Inc00152 overexpression promoted epithelial-mesenchymal transition (EMT) and increased neuropilin-1 (NRP1) expression in the CRC cells. By contrast, lnc00152 silencing exerted a counteractive effect. Collectively, these findings demonstrate the critical role of Inc00152 in tumor growth and progression in CRC,
\end{abstract}

Correspondence to: Dr Jie Cao, Department of Gastrointestinal Surgery, Guangzhou Digestive Disease Center, Guangzhou First People's Hospital, Guangzhou Medical University, 1 Panfu Road, Guangzhou, Guangdong 510180, P.R. China

E-mail: xrd588@163.com

${ }^{*}$ Contributed equally

Key words: colorectal cancer, lncRNA 00152, miRNA-206, neuropilin-1, proliferation and invasion and identify a novel therapeutic target associated with CRC development and progression.

\section{Introduction}

Colorectal cancer (CRC), one of the most common types of cancer, is associated with a high mortality rate worldwide (1). While the diagnosis of and treatment strategies for CRC have improved, the overall survival of patients with CRC remains unsatisfactory (2). Current CRC treatment includes neoadjuvant radiotherapy and chemotherapy (3), surgical therapy $(4,5)$ and immunization therapy $(6,7)$. In the majority of cases, however, CRC is diagnosed at an advanced pathological stage before any symptoms appear, resulting in poor survival rates. Recent studies have indicated that long non-coding RNAs (lncRNAs) are critical factors in regulating the development, differentiation and apoptosis (8-10) of cancer cells. IncRNAs are also associated with tumor progression and metastasis (11). In CRC specifically, lncRNA could inhibit tumor growth by deactivating Akt signaling (12). The aberrant expression of lncRNAs is associated with a poor survival (13), lymph node metastasis (12) and the modulation of pithelial-mesenchymal transition (EMT) in CRC (14). Therefore, lncRNAs may function as novel therapeutic targets and molecular markers for the diagnosis of CRC. Several studies have identified molecular markers affecting CRC dynamics (15-17). For instance, miRNA-126 expression has been shown to exert a critical effect on CRC pathogenesis (18). However, further clarifications of the systemic correlation between miRNAs and $\operatorname{lncRNAs}$ are required.

The IncRNA-miRNA complex forms a regulatory network in non-small cell lung cancer A549 cells (19), indicating that IncRNAs are involved in controlling tumor progression by interacting with miRNAs. Additional molecules, such as the orphan nuclear receptors, Nur77, RAR $\gamma$ and WNT, signal the inhibitors, OVOL2, Myb-like SWIRM and MPN domains 1 (MYSM1), which influence the invasion, metastasis and progression of CRC (14,20-22). Some bioactive enzymes, such as serum $\beta$-glucuronidase, are also considered potential markers of CRC (23). Neuropilin-1 (NRP1) is a transmembrane glycoprotein that can function as an oncogene by participating 
in the development and progression of various types of cancer (24,25), including CRC (26). Numerous biomarkers have been identified in CRC cells; however, there is still a need for further clarification of the mechanisms driving the regulation between biomarkers and tumor progression and metastasis.

In this study, we found that IncRNA 00152 (Inc00152) was overexpressed in CRC tumors and cancer cells when compared to adjacent non-tumor tissues. Inc00152 is an important factor affecting cell proliferation and invasion in vitro and tumor growth in vivo. The regulation of lnc00152 altered NRP1 expression and affected EMT by modulating $\mathrm{N}$-cadherin and E-cadherin expression. Finally, we found that the effects of lnc00152 were regulated by miRNA-206. Our findings indicate that $\operatorname{lnc} 00152$ may function as a therapeutic target against CRC.

\section{Materials and methods}

Ethics statements. The use of human samples was approved by the Ethics Committee Guangzhou First People's Hospital and all patients involved in the study provided written informed consent. The use of animals in this study was approved by the Ethics Committee of the Laboratory Animal Center of South China University of Technology.

Patients and tissue samples. This study was conducted using a total of 80 paraffin-embedded CRC samples and 40 adjacent non-tumor tissue samples. All the samples were histopathologically and clinically diagnosed at Guangzhou First People's Hospital from 2010 to 2016. None of the recruited participants had received adjunctive treatment prior to surgery. The histological characterization and clinicopathological staging of the samples were determined according to the Union for International Cancer Control (UICC). The clinicopathological information was available for all samples (Table I). The CRC specimens and the matched adjacent non-cancerous tissues were frozen and stored in liquid nitrogen until further use. The overall survival (OS) was defined as the period between diagnosis and either death or the last follow-up. Follow-up ranged from 0 to 78 months. The Kaplan-Meier overall survival curve was obtained according to lnc00152 expression and the cumulative survival ratio was calculated at different time-points during follow-up.

Cell culture. We obtained the CRC cell lines LoVo, SW480 and SW620 from The Cell Bank of Type Culture Collection of Chinese Academy of Sciences (Shanghai, China). The cells were grown at $37^{\circ} \mathrm{C}$ with $5 \% \mathrm{CO}_{2}$ in RPMI-1640 medium (Invitrogen, Carlsbad, CA, USA) supplemented with $10 \%$ fatal bovine serum (FBS) (Invitrogen), $100 \mathrm{U} / \mathrm{ml}$ penicillin and $100 \mu \mathrm{g} / \mathrm{ml}$ streptomycin (Invitrogen).

$R N A$ extraction and reverse transcription (real-time) quantitative PCR (RT-qPCR). RT-qPCR was used to determine the expression of lnc00152. We extracted total RNA from the tissues and cultured cells using TRIzol reagent (Invitrogen/ Life Technologies) according to the manufacturer's instructions. Subsequently, $2 \mu \mathrm{g}$ of the extracted total RNA was subjected to reverse transcription (RT) using a reverse transcription kit (Cat. no. 639505; Takara, Tokyo, Japan). In order to perform real-time (quantitative) PCR, we used the One Step SYBR-Green ${ }^{\circledR}$ (Tokyo, Japan) PrimeScript ${ }^{\mathrm{TM}}$ RT-PCR kit (Cat. no. RR064A; Takara). For real-time fluorescence detection we used the ABI7300 real-time PCR thermal cycle instrument (Applied Biosystems, Foster City, CA, USA) according to the manufacturer's instructions. The $20 \mu \mathrm{l}$ total reaction mixture contained 20 ng cDNA, $10 \mu \mathrm{l}$ of $2 \mathrm{X}$ SYBR-Green master mix, $200 \mathrm{nM}$ of forward and reverse primers and PCR-grade water. The PCR cycling conditions were set to $95^{\circ} \mathrm{C}$ for $3 \mathrm{~min}$, followed by 35 cycles of $95^{\circ} \mathrm{C}$ for $30 \mathrm{sec}$, and $55^{\circ} \mathrm{C}$ for $30 \mathrm{sec}$. A melting curve analysis verified the specificity of each primer after PCR to ensure amplification specificity. Relative gene expression was calculated using the $2^{-\Delta \Delta \mathrm{Cq}}$ method (27), and we normalized the results to the expression of 18s rRNA. For miRNA-206 expression detection, we validated human U6 as the normalizer. The sequences of the primers used for PCR are presented in Table II.

Transfection with small interfering RNA (siRNA) targeting lnc00152. We selected the site in the $\beta$-catenin mRNA sequence as a siRNA target. Three targeted siRNAs against lnc00152 (lnc00152-siRNAs) were created and the sequences were as follows: siRNA1, GGGAAATAAATGACTGGAT; siRNA2, GGAGATGAAACAGGAAGCT; and siRNA3, GGGAATGG AGGGAAATAAA. All the siRNAs, as well as the negative control siRNA were synthesized and purified by RiboBio Biotech Corp. (Guangzhou, China). The siRNA plasmids were transfected into the SW620 cells with Lipofectamine 2000 (Invitrogen) at a working concentration of $200 \mathrm{nM}$ and incubated for $24 \mathrm{~h}$. After the incubation period, the cells were harvested for use in RT-qPCR analysis.

Inc00152 overexpression, plasmid construction and transfection. The full-length lnc00152 was synthesized by Shanghai Sangon Biotech Corp. (Shanghai, China) and cloned into pLV4 plasmids (Promega Corp., Madison, WI, USA). Empty pLV4 plasmids, without the insertion, served as the negative control. Cell transfection was conducted with Lipofectamine 2000 reagent according to the manufacturer's instructions. Further analyses were conducted at $24 \mathrm{~h}$ following transfection.

Cell Counting kit-8 (CCK-8) assay. A Cell Counting kit-8 assay ('CCK-8', KeyGen Biotech, Nanjing, China) was used to evaluate cell viability at $12,24,36,48,60$ and $72 \mathrm{~h}$ of culture. Briefly, both the control and infected cells were seeded at a density of $2 \times 10^{3}$ cells/well into a 96-well plate, to which $10 \mu \mathrm{l}$ of the $10 \%$ CCK- 8 solution was subsequently added. The plate was incubated at $37^{\circ} \mathrm{C}$ for $4 \mathrm{~h}$ in a $5 \% \mathrm{CO}_{2}$ incubator. A microplate reader (Spectramax M5; Molecular Devices Corp., San Jose, CA, USA) was used to measure the product absorbance at $490 \mathrm{nM}$, and the results are reported as follows: (OD value of sample - OD value of blank)/(OD value of blank). Data were collected from 3 independent experiments.

Transwell invasion assay. A Transwell invasion assay was used to evaluate the invasive potential of the transfected cells. We used Matrigel invasion chambers (Corning Inc., Corning, NY, USA) with $8-\mu$ m pores (BD Biosciences, San Jose, CA, USA). In brief, a concentration of $2 \times 10^{4}$ cells $/ 100 \mu 1$ was re-suspended in DMEM without fetal bovine serum (FBS) then subsequently seeded on top of a Matrigel-coated Transwell. A total 
Table I. Detailed clinical information of the patients with colorectal cancer.

\begin{tabular}{|c|c|c|c|}
\hline \multirow[b]{2}{*}{$\begin{array}{l}\text { Clinical } \\
\text { characteristics }\end{array}$} & \multicolumn{3}{|c|}{ Patients } \\
\hline & $\begin{array}{c}\text { No. of } \\
\text { patients }\end{array}$ & $\begin{array}{c}\text { IncRNA } 00152 \\
\text { expression }\end{array}$ & $\mathrm{P}$-value \\
\hline \multicolumn{4}{|l|}{ Age (years) } \\
\hline$\geq 60$ & 60 & $288.76 \pm 150.94$ & 0.968 \\
\hline$<60$ & 20 & $321.38 \pm 129.96$ & \\
\hline \multicolumn{4}{|l|}{ Sex } \\
\hline Male & 43 & $295.75 \pm 152.82$ & 0.563 \\
\hline Female & 37 & $298.27 \pm 139.44$ & \\
\hline \multicolumn{4}{|c|}{ Pathological grade } \\
\hline I-II & 37 & $222.71 \pm 83.22$ & 0.008 \\
\hline III-IV & 43 & $360.77 \pm 158.31$ & \\
\hline \multicolumn{4}{|l|}{ Tumor invasion } \\
\hline $\mathrm{T}_{1}-\mathrm{T}_{2}$ & 19 & $253.38 \pm 111.75$ & 0.383 \\
\hline $\mathrm{T}_{3}-\mathrm{T}_{4}$ & 61 & $316.08 \pm 150.66$ & \\
\hline \multicolumn{4}{|l|}{$\begin{array}{l}\text { Lymph-node } \\
\text { metastasis }\end{array}$} \\
\hline $\mathrm{N}_{0}$ & 39 & $227.72 \pm 84.06$ & 0.004 \\
\hline $\mathrm{N}_{1}-\mathrm{N}_{2}$ & 41 & $362.73 \pm 161.88$ & \\
\hline \multicolumn{4}{|c|}{ Distant metastasis } \\
\hline $\mathbf{M}_{0}$ & 60 & $279.70 \pm 144.43$ & 0.429 \\
\hline $\mathrm{M}_{1}$ & 20 & $348.55 \pm 141.26$ & \\
\hline \multicolumn{4}{|c|}{ Vascular invasion } \\
\hline No & 65 & $290.74 \pm 155.12$ & 0.142 \\
\hline Yes & 15 & $323.67 \pm 95.55$ & \\
\hline
\end{tabular}

Numbers in bold indicate statistical significance $(\mathrm{P}<0.05)$.

of $600 \mu \mathrm{l}$ of DMEM containing $10 \%$ FBS was added to the lower chambers. Following a 24 -h incubation period, the filters separating the upper and lower chamber were washed twice with PBS, fixed by methanol and stained with crystal violet at room temperature for $10 \mathrm{~min}$ (Beijing Solarbio Science \& Technology Co. Ltd., Beijing, China). We then counted the number of stained cells under a light microscope (Nikon Eclipse E200; Nikon, Tokyo, Japan; magnification, x100). The area of each membrane was, on average, 5 visual fields. This experiment was repeated independently, in triplicate.

Establishment of tumor xenograft models. We purchased 12 athymic nude mice (6 males and 6 females; weighing 18-20 g, 4 weeks old) from Beijing Slac Laboratory Animal Co. Ltd. (Beijing, China), which we housed in high-efficiency particulate air-filtered cages in a pathogen-free facility. The housing environment was maintained at $25 \pm 2{ }^{\circ} \mathrm{C}, 45-55 \%$ humidity, and a standard 12-h dark/12-h light cycle, and we fed mice an autoclaved diet with free access to water. We cleaned and sterilized the inoculation area (right upper limb) with ethanol and iodine solutions before subcutaneously injecting the SW620 cells $\left(1 \times 10^{6} / \mathrm{ml}\right)$ transfected with pLV-NC or pLV-lnc00152 into the mice ( $n=6$ mice per group). Tumor
Table II. Sequences of primers used in RT-qPCR.

\begin{tabular}{ll}
\hline Gene name & \multicolumn{1}{c}{ Primer sequence (5'-3') } \\
\hline lnc00152 & F: CCACCAGCCTCTCCTTGAAT \\
& R: GGCTGAGTCGTGATTTCGGT \\
E-cadherin & F: TGCCGCCATCGCTTACACCATC \\
& R: GGTCAGCAGCTTGAACCACCAG \\
N-cadherin & F: CCCACAGCTCCACCATATGACTC \\
& R: CCTGCTCACCACCACTACTTGAG \\
Human U6 & F: CTCGCTTCGGCAGCACA \\
& R: AACGCTTCACGAATTTGCGT \\
NRP1 & F: GAGGCATGAAGGCAGACAGAG \\
& R: GAGGCATGAAGGCAGACAGAG \\
18s rRNA & F: CCTGGATACCGCAGCTAGGA \\
& R: GCGGCGCAATACGAATGCCCC \\
miRNA-206 & F:ACACTCCAGCTGGGTGGAATGTAAG \\
& GAAGTGTG \\
& R: GCAGGGTCCGAGGTATTCG
\end{tabular}

volumes $\left(0.5 \times\right.$ length $\mathrm{x}$ width $\left.{ }^{2}\right)$ were measured on days 10 , 14, 18, 22 and 26 following implantation. Of note, the largest tumor diameter of a single tumor in our study was $18 \mathrm{~mm}$ and the largest volume was $570.317 \mathrm{~mm}^{3}$. No mouse developed multiple tumors. After 4 weeks, the mice were sacrificed by CO2 inhalation (20\% of the chamber volume was displace per minute by the flow of $\mathrm{CO}_{2}$ ). Tumor tissues were excised, then fixed in a $4 \%$ paraformaldehyde solution for further analysis. All the animal experiments were performed in the Animal Laboratory Center of Guangzhou First Hospital.

Western blot analysis. For protein extraction, the cultured cells were lysed in a cell lysis buffer containing $140 \mathrm{mM} \mathrm{NaCl}, 10 \mathrm{mM}$ Tris- $\mathrm{HCl}, 1 \%$ Triton $\mathrm{X}-100,1 \mathrm{mM}$ EDTA and $1 \mathrm{X}$ protease inhibitor. The tumor tissues were homogenized in lysis buffer (pH 7.5) containing $300 \mathrm{mM} \mathrm{NaCl}, 50 \mathrm{mM}$ Tris- $\mathrm{HCl}, 0.5 \%$ Triton X-100 and 1X protease inhibitor, and then incubated at $4^{\circ} \mathrm{C}$ for $30 \mathrm{~min}$. The cell and tissue lysates were centrifuged at $3,000 \mathrm{x} \mathrm{g}$ at $4^{\circ} \mathrm{C}$ for $15 \mathrm{~min}$. We used the BCA method to determine the protein concentration (Sangon, Shanghai, China). Subsequently, $10 \mu \mathrm{g}$ aliquots of the cell and tissue lysates were loaded onto each lane of a $10 \%$ polyacrylamide gel, then blotted onto polyvinylidene difluoride (PVDF) membranes. After blocking with a PBST containing 5\% non-fat dry milk, the membranes were incubated with antibodies against NRP1 (1:500; Cat. no. ab81321; Abcam, Cambridge, MA, USA), N-cadherin (1:500; Cat. no. ab18203; Abcam), E-cadherin (1:500; Cat. no. cst9961, 1:1,000; Cell Signaling Technology, Danvers, MA, USA) and GAPDH (1:1,000; Cat. no. 8245; Abcam). The membranes were then washed with TBST 3 times, then incubated them with peroxidase-linked anti rabbit IgG secondary antibody (Cat. no. A16096; Thermo Fisher Scientific, Waltham, MA, USA) for $1 \mathrm{~h}$ at room temperature. These proteins were visualized by using an ECL western blotting detection kit (Amersham Biosciences, Piscataway, NJ, USA). This experiment was repeated in triplicate 
A
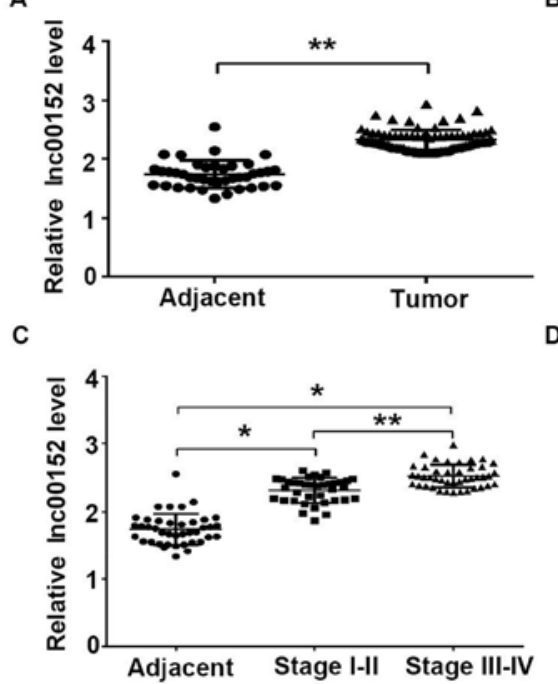

E

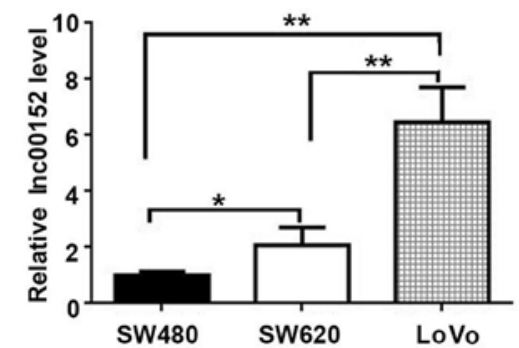

B
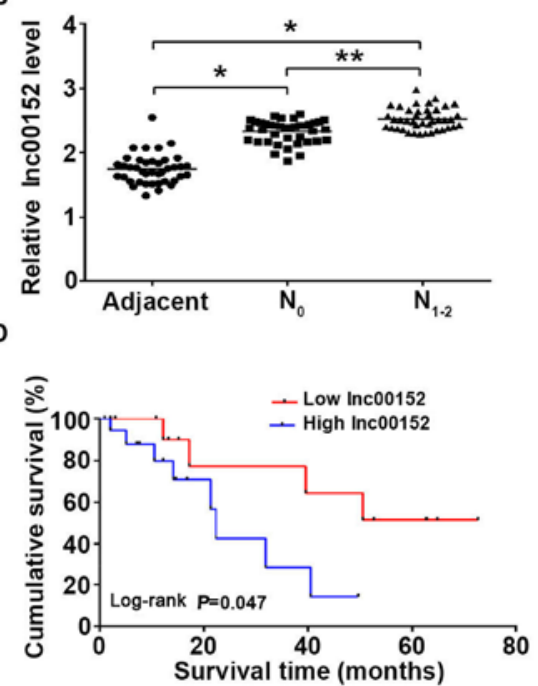

$\mathbf{F}$

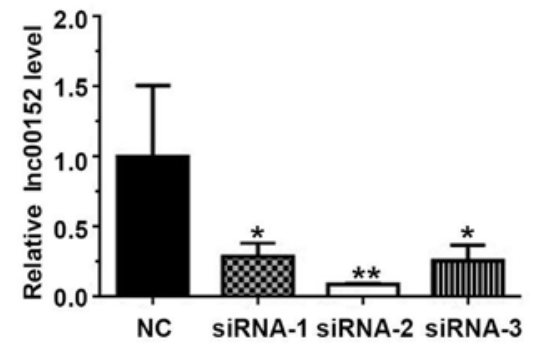

Figure 1. Relative lnc00152 expression in colorectal tumor tissues, cancer cells and its clinical significance. (A) Relative expression of lnc00152 expression was examined by RT-qPCR and normalized to 18s rRNA. Inc00152 expression was higher in the tumor tissues $(\mathrm{n}=80)$ than in the adjacent non-tumor tissues $(\mathrm{n}=40)$. (B) The association between lnc00152 expression and lymph-node metastasis indicated that tumors with lymph-node metastasis had higher expression levels of $1 n c 00152$. (C) Tumors at an advanced pathological stage also exhibited higher levels of lnc00152. (D) Kaplan-Meyer overall survival curves display higher lnc00152 levels in patients with colorectal cancer (CRC) with lower survival times (log-rank test, $\mathrm{P}=0.047$ ). (E) Relative lnc00152 expression in CRC cell lines (SW480, SW620 and LoVo) was determined by RT-qPCR; differential expression levels of lnc00152 were observed in the 3 CRC cell lines. (F) siRNAs targeting lnc00152 effectively downregulated the lnc 00152 levels in the $\mathrm{SW} 620$ cells. ${ }^{*} \mathrm{P}<0.05,{ }^{* *} \mathrm{P}<0.01$ vs. NC group or as indicated.

independently. Visualization of proteins and band intensity was determined using ImageJ software version $14.8(\mathrm{NIH}$ MD, USA). The gray level was analyzed using Quantity One software (Bio-Rad, Hercules, CA, USA).

Hematoxylin and eosin $(H \& E)$ staining. The tumor xenograft sections were deparaffinized in xylene and dehydrated in alcohol. The sections were then stained in Harris' hematoxylin solution for $8 \mathrm{~min}$ before bluing in $0.2 \%$ ammonia water or saturated lithium carbonate solution for $1 \mathrm{~min}$. After rinsing in $95 \%$ alcohol, the sections were counterstained in eosinphloxine solution for $1 \mathrm{~min}$. Finally, we mounted sections with a xylene-based medium and visualized the slides with a Nikon ECLIPSE 90i (Nikon; magnification, x100). Two pathologists evaluated the images in a blinded manner.

Immunohistochemistry. The tumor xenograft sections were washed in PBS and blocked for 60 min in $0.3 \%$ Triton X-100 and PBS with $5 \%$ bovine serum albumin, before being incubated overnight at $4^{\circ} \mathrm{C}$ with anti-E-cadherin (1:50; Cat. no. 9961) or anti-N-cadherin (1:50; Cat. no. 4061) antibodies (Cell Signaling Technology). We then applied HRP-conjugated secondary antibody (1:2,000; Cat. no. 7074; Abcam, Cambridge, UK) to the slides before a $1 \mathrm{~h}$ room temperature incubation. To develop color on the slide, we added diaminobenzidine (DAB) $/ \mathrm{H}_{2} \mathrm{O}_{2}$ before the slides were visualized with a Nikon ECLIPSE 90i microscope (Nikon; magnification, x100). As mentioned above, two pathologists evaluated the images in a blinded manner.

Site-directed mutagenesis and dual-luciferase reporter assay. We used a SQE-PCR to perform site mutation on lnc00152 and then ligated the $\operatorname{lnc} 00152$ insert into a psiCHEK $^{\mathrm{TM}}-2$ vector (Promega Corp.). Three fragments were cloned by PCR using the following primers, including Psi-152F, CCGCTC GAGCATCAT TGGGAATGGAGGGAAAT;Psi-152R,ATTTGCGGCCGCTT CTGTTTTCTTTAGTTTTGCTT; Mut-152F1, GTTTCAAAT TGGAGCCTTCGACAAGCGGTGCCTGAGC; Mut-152R1, CACCGCTTGTCGAAGGCTCCAATTTGAAACTTAAAA AGC; Mut-152F2, GCCTCCATCCGAGCCTTCACCTCCGTC TGCATCCCTCG; and Mut-152R2, AGACGGAGGTGAAGG CTCGGATGGAGGCTGGCAAGTTTC. Human 293T cells (Cell Bank of Type Culture Collection of Chinese Academy of Sciences, Shanghai, China) were co-transfected with $150 \mathrm{ng}$ of miRNA-206 mimics, miRNA NC mimics, miRNA-206 inhibitors and miRNA-NC inhibitor (RiboBio). Subsequently, $50 \mathrm{ng}$ of wild-type lnc00152 or lnc00152 mutant fluorescent vector were co-transfected with the psiCHEK ${ }^{\mathrm{TM}}-2$ vector into the human 293T cells using Lipofectamine 2000 (Invitrogen). We used miRNA-NC as a negative control and repeated the transfections in triplicate. Luciferase assay was conducted at 
A

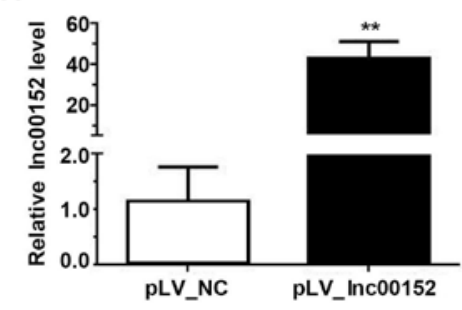

C

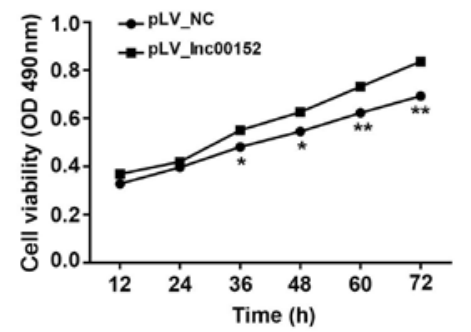

B

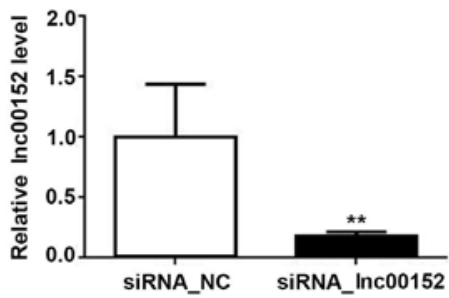

D

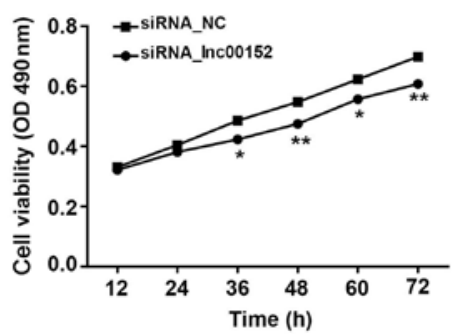

E
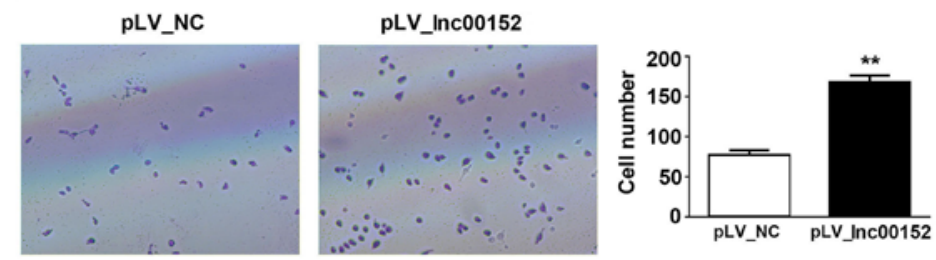

F SIRNA_NC

SiRNA_Inc00152
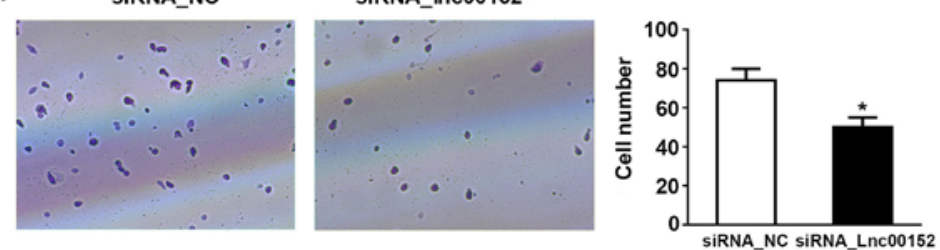

Figure 2. A high lnc00152 expression enhances colorectal cancer (CRC) cell viability and invasion. (A) SW620 cells were transfected with pLV_NC and pLV_lnc00152. The efficiency of the lnc00152 overexpression plasmid was evaluated by RT-qPCR. (B) The SW620 cells were transfected with lnc00152 siRNA-2 or siRNA-NC. The efficiency of the siRNA-lnc00152 construct was evaluated by RT-qPCR. (C) A CCK-8 assay was used to examine changes in cell viability. Inc00152 overexpression enhanced SW620 cell viability. (D) A CCK-8 assay was used to examine changes in cell viability. Inc00152 silencing impaired SW620 cell viability. (E) A Transwell invasion assay was used to examine changes in cell invasiveness. Inc00152 overexpression promoted the invasive ability of the SW620 cells. (F) lnc00152 silencing suppressed SW620 cell invasion. ${ }^{*} \mathrm{P}<0.05,{ }^{* *} \mathrm{P}<0.01$ vs. pLV_NC.

$48 \mathrm{~h}$ following transfection, and we normalized the relative luciferase activity using the luciferase assay kit (Promega Corp.) at $48 \mathrm{~h}$ after transfection. Finally, to elucidate the interaction between lnc00152 and miRNA-206, we expressed miRNA inhibitor and mimics into the 293T cells and subsequently applied qPCR analysis for miRNA-206 and lnc00152 expression at $48 \mathrm{~h}$ following transfection.

Statistical analysis. Summarized data are presented as the means \pm SEM. We performed all statistical analyses using SPSS19.0 software (SPSS Inc., Chicago, IL, USA). A Chi-square test was used to analyze the association between lnc00152 expression and the patient clinicopathological characteristics. Survival curves were plotted by the Kaplan-Meier method and compared using the log-rank test. Comparisons between 2 groups for statistical significance were carried out with two-tailed paired Student's t-tests. For analyses involving multiple sample groups, statistical significance was determined using one-way ANOVA followed by Tukey's test for multiple comparisons. Statistical significance was set at a P-value $<0.05$.

\section{Results}

High expression of lnc00152 is associated with low survival rates of patients with $C R C$. In order to determine the potential role of lnc00152 in CRC, we first compared lnc00152 expression levels between CRC tissues and adjacent non-tumor tissues. The lnc00152 expression level was significantly higher in the CRC tissues compared with the adjacent non-tumor tissues (Fig. 1A, P<0.01). In terms of lymph-node metastasis, the lnc00152 expression level was higher in the $\mathrm{N}_{1-2}$ grade tumors than in the $\mathrm{N}_{0}$ grade tumors (Fig. $1 \mathrm{~B}, \mathrm{P}<0.01$ ). Furthermore, both the $\mathrm{N}_{1-2}$ and $\mathrm{N}_{0}$ grade tumors exhibited significantly higher levels of lnc00152 than the adjacent non-tumor tissues (Fig. 1B, $\mathrm{P}<0.05)$. Similarly, the lnc00152 level was associated with the pathological grade of colorectal tumors. The malignancy of CRC was associated with the level of lnc00152 expression, with significantly higher levels observed in stage III-IV than in stage I-II tumors (Fig. 1C, P<0.01). Furthermore, the lnc00152 levels were associated with the overall survival, with significantly shorter survival times observed in patients with CRC 
A
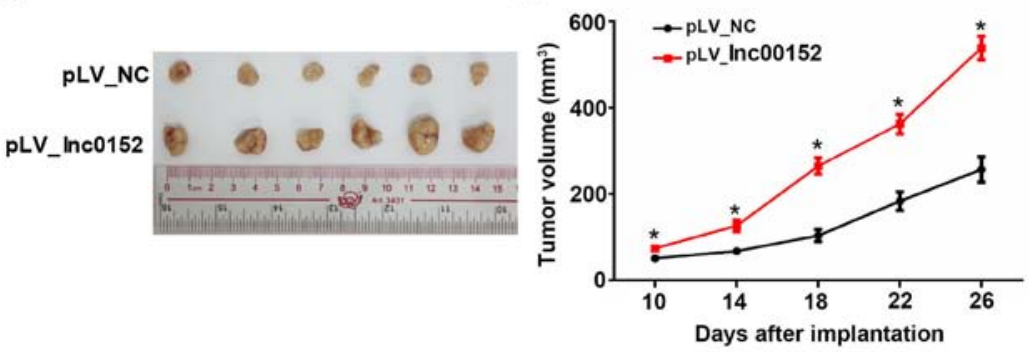

D

c
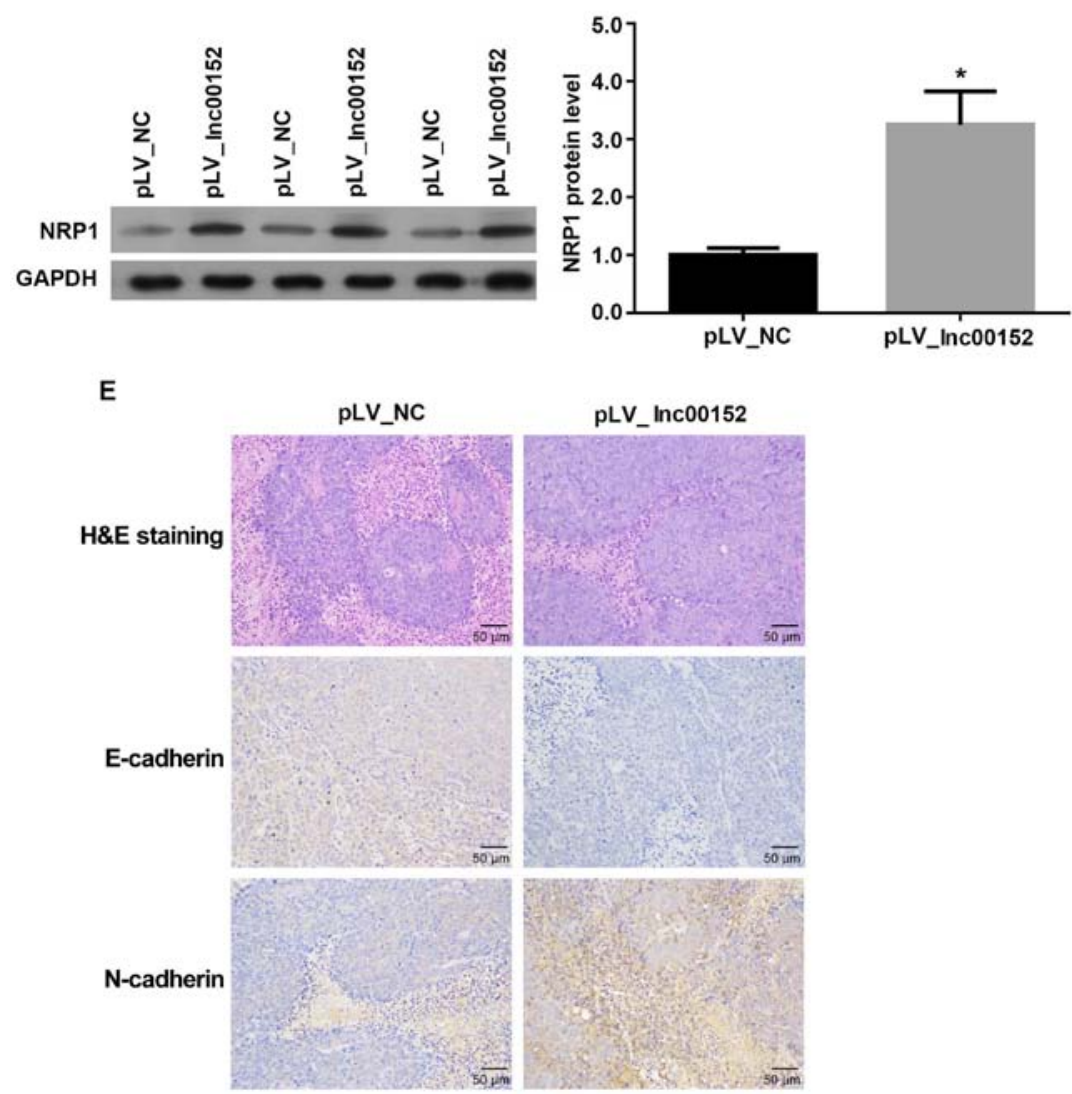

Figure 3. Inc00152 overexpression promotes tumor growth in vivo. (A) The tumors were separated from nude mice following the injection of SW620 cells transfected with pLV_NC or pLV_Lnc00152 ( $\mathrm{n}=6$ per group). (B) Tumor volume curves were measured on days 10, 14, 18, 22 and 26 following implantation. Tumor volume significantly increased in the lnc00152-overexpressing (pLV_Lnc00152 transfection) group. (C and D) Western blot analysis of NRP1 expression in tissues of resected tumors. lnc00152 overexpression led to an increased NRP1 level. (E) Results of immunohistochemistry indicated that lnc00152 overexpression downregulated E-cadherin expression and upregulated N-cadherin expression. Upper panels, H\&E staining; lower panels, immunostaining of E-cadherin and $\mathrm{N}$-cadherin. Scale bar, $50 \mu \mathrm{m}$. ${ }^{*} \mathrm{P}<0.05$.

with higher lnc00152 levels (Fig. 1D and Table I, log-rank test, $\mathrm{P}=0.047)$. Neither age nor sex were significantly associated with lnc00152 expression ( $\mathrm{P}=0.968$ and $\mathrm{P}=0.563$, respectively); however, lnc00152 expression was significantly associated with the pathological grade $(\mathrm{P}=0.008)$ and lymph-node metastasis $(\mathrm{P}=0.004)$. The human CRC cells (SW480, SW620 and LoVo) also expressed lnc00152, with the highest expression observed in the LoVo cells (Fig. 1E). These results suggest that lnc00152 overexpression is an indicator of CRC in human tissues and cells, and it may be useful in the prognosis of CRC.

Inc00152 enhances the proliferative and invasive ability of the $C R C$ cells in vitro. To elucidate the mechanisms through which lnc00152 regulates CRC growth, we controlled the expression level of lnc00152 in the CRC cancer cell line, SW620. Inc00152 expression was significantly decreased in the siRNA-transfected cells, with an optimal silencing effect observed with siRNA-2 ( $\mathrm{P}<0.01$, Figs. 1F and 2B). Additionally, we established that the expression of linc00152 in the SW620 cells was significantly higher in the cells transfected with the lnc 00152 overexpression vector compared with the pLV-NC-transfected cells (Fig. 2A, $\mathrm{P}<0.01)$. The overexpression of 1 c 00152 enhanced the viability of the SW620 cells at 36, 48, 60 and $72 \mathrm{~h}$ following transfection (Fig. 2C). The silencing of lnc00152, however, significantly decreased cellular proliferation (Fig. 2D) at $36(\mathrm{P}<0.05), 48$ $(\mathrm{P}<0.01), 60(\mathrm{P}<0.05)$, and $72 \mathrm{~h}(\mathrm{P}<0.01)$ following transfection, but not at 12 or $24 \mathrm{~h}$. From the Transwell assay evaluating the cell invasive potential, we found that the overexpression of $\ln 00152$ enhanced the invasive ability of the SW620 cells (Fig. 2E, P<0.01). Conversely, lnc00152 silencing decreased the 
A

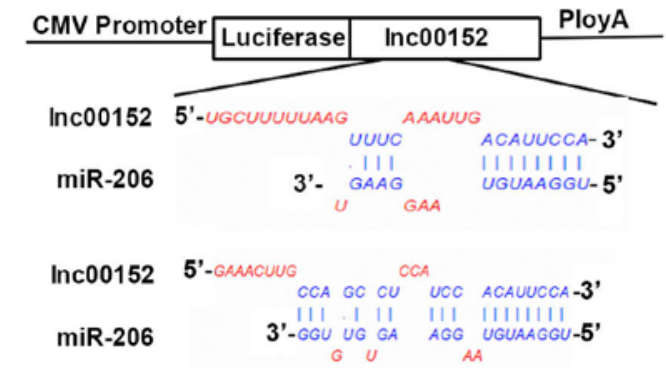

C

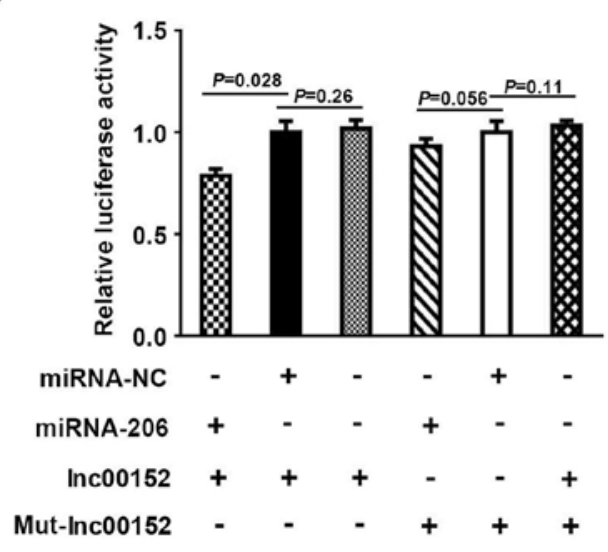

D

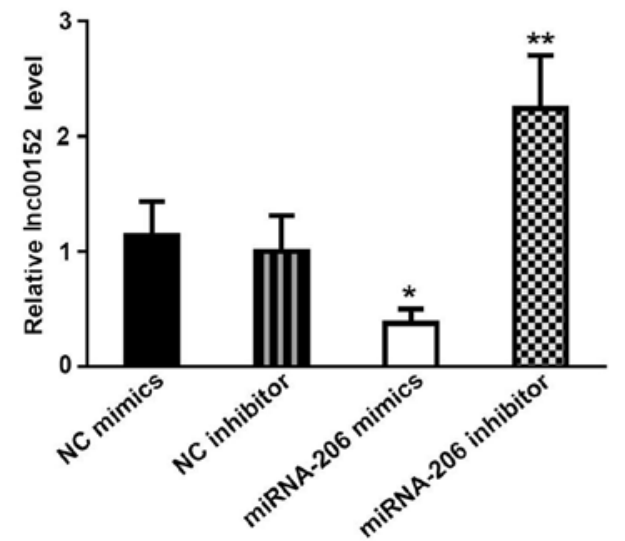

B
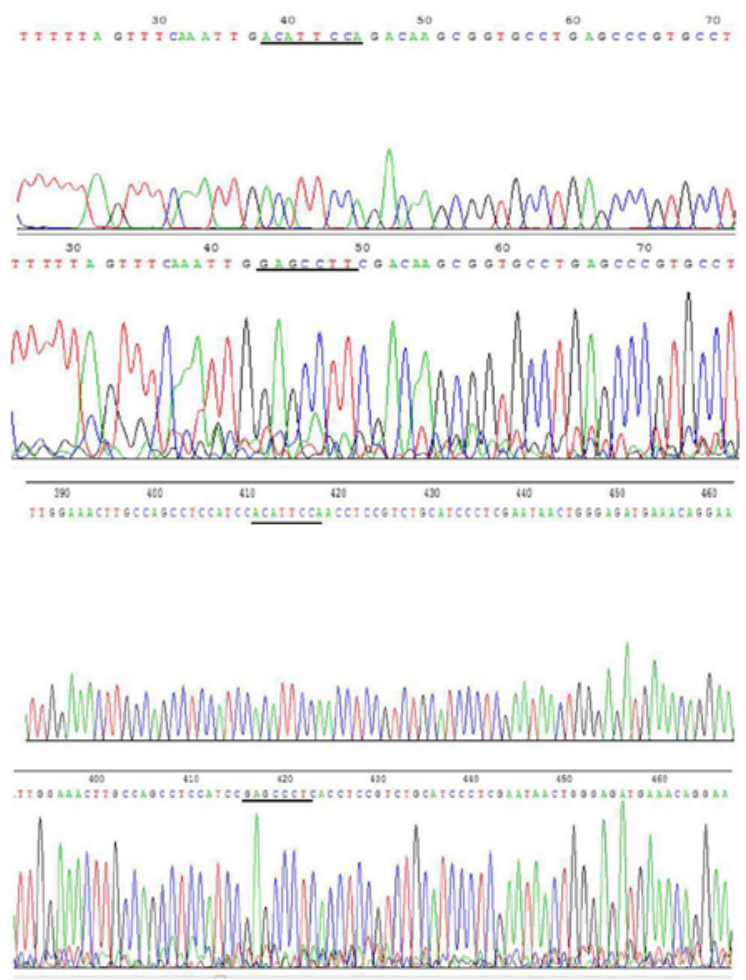

E

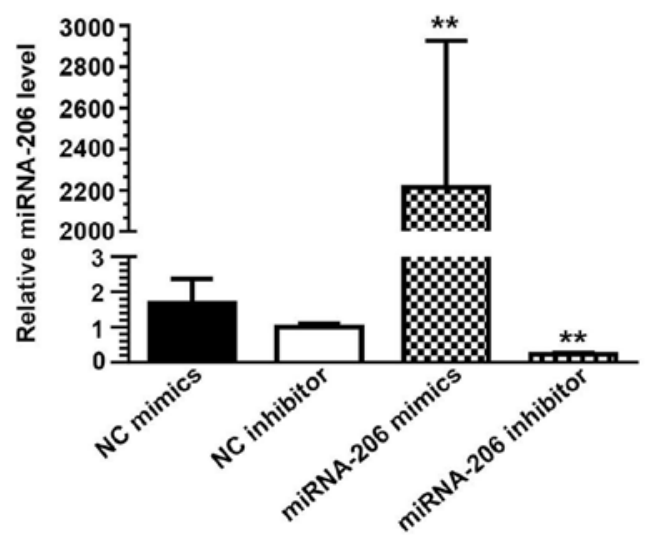

Figure 4. Effects of lnc00152 on colorectal cancer cells are regulated by miRNA-206. (A) Schematic diagram showing the interaction between lnc00152 and miRNA-206. (B) Sequence of mutant lnc00152. (C) Luciferase assay showing the specific interaction between lnc00152 and miRNA-206. (D) The regulation of miRNA-206 altered the Inc00152 level. (E) The miRNA-206 expression level was affected by transfection with miRNA-206 mimics and inhibitor. ${ }^{*} \mathrm{P}<0.05$, ${ }^{* *} \mathrm{P}<0.01$.

number of invading cells relative to those transfected with the control siRNA (siRNA_NC) (Fig. 2F, P<0.05). Consequently, our findings indicate that lnc00152 enhances the proliferative and invasive ability of CRC cells.

Inc00152 overexpression enhances colorectal tumor growth in vivo. To determine whether lnc00152regulates tumor growth in vivo, we xenografted lnc00152 stably expressing SW620 cells into nude mice. The volumes of tumors derived SW620 cells overexpressing lnc00152 were significantly greater than those of tumors derived from the pLV-NC-transfected control cells from days 10 to 26 following implantation (Fig. $3 \mathrm{~A}$ and $\mathrm{B}, \mathrm{P}<0.05$ ). Additionally, the protein levels of NRP1 were increased in tumors derived from the lnc00152-overexpressing cells (Fig. 3C and $\mathrm{D}, \mathrm{P}<0.05)$. Furthermore, lnc00152 overexpression also promoted EMT in the SW620-derived tumors by decreasing the level of E-cadherin, while increasing $\mathrm{N}$-cadherin expression (Fig. 3E). H\&E staining also confirmed the cellular alteration in lnc00152-overexpressing colorectal tumors, which exhibited typical morphological characteristics of more malignant tumors (large cell morphology, large cell nucleus and malformed nuclei). Collectively, these data indicate that lnc00152 overexpression promotes tumor growth and EMT in vivo.

miRNA-206 is a target gene of lnc00152. A dual luciferase assay verified the target gene of miRNA-206 as 1nc00152. Plasmid construction and sequence data and the sequence of mutant lnc00152 are described in Fig. 4A and B. When the 


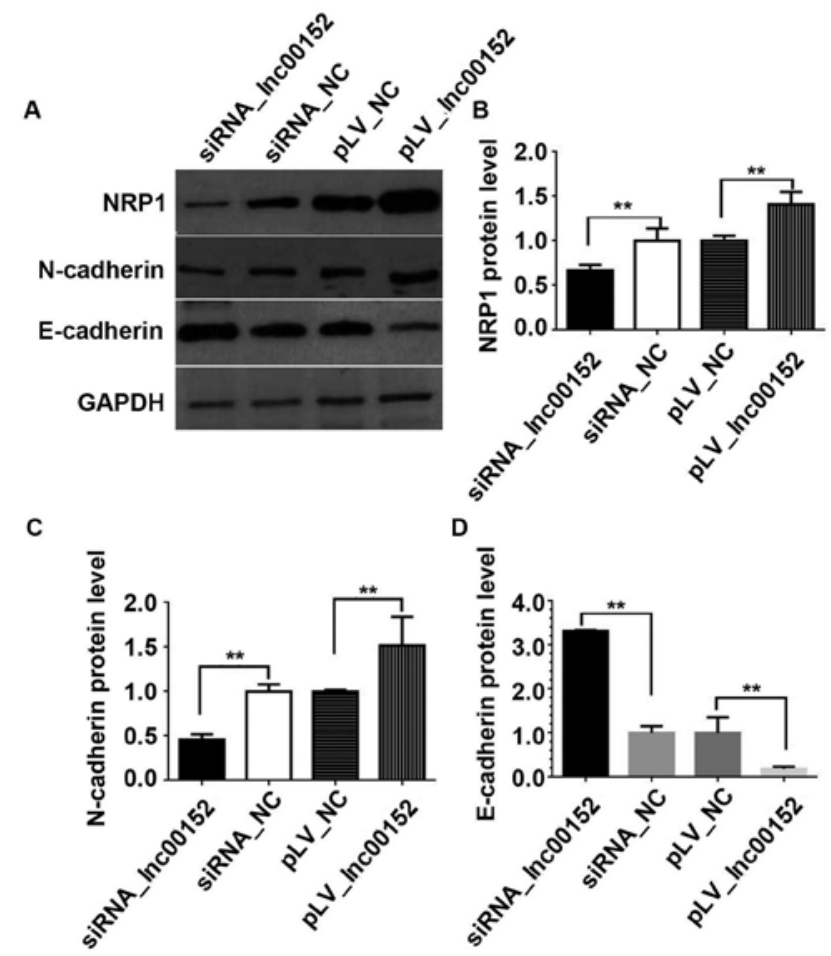

Figure 5. Regulation of 1 nc00152 affects NRP1 expression and epithelialmesenchymal transition in colorectal cancer (CRC) cells. (A) Western blot analysis of NRP1, E-cadherin and N-cadherin expression following the transfection of human 293T cells transfected with siRNA_NC, siRNA_Lnc00152, pLV_NC, or pLV_Lnc00152. The regulation of Inc00152 expression altered the expression level of (B) NRP1, (C) N-cadherin, and (D) E-cadherin. *** $\mathrm{P}<0.01$.

SW620 cells were transfected with the lnc00152 overexpression vector together with miRNA-NC, the relative luciferase activity did not differ significantly from that in the cells transfected with the 1 nc 00152 overexpression vector only (Fig. 4C, $\mathrm{P}=0.26>0.1$ ). By contrast, the relative luciferase activity decreased when the cells were transfected with the lnc00152 overexpression vector and the miRNA-206 mimic (Fig. $4 \mathrm{C}, \mathrm{P}=0.028<0.05$ ), demonstrating a direct interaction between 1 nc00152 and miRNA-206. Co-transfection with mutated lnc00152 (Mut-Lnc00152) and miRNA-206 did not result in reduced relative luciferase activity (Fig. $4 \mathrm{C}, \mathrm{P}>0.05$ ), indicating that mutated $1 \mathrm{nc} 00152$ disrupted the interaction between $\operatorname{lnc} 00152$ and miRNA-206. In addition, the 1 nc 00152 level was lower in the cells transfected with miRNA-206 mimics compared to those transfected with NC mimics (Fig. 4D, P<0.05), while the lnc00152 level increased significantly following incubation with the miRNA-206 inhibitor compared to the $\mathrm{NC}$ inhibitor (Fig. 4D, $\mathrm{P}<0.01$ ). In the presence of miRNA-206 mimics, the miRNA-206 level increased significantly (Fig. 4E, P<0.01), while it decreased significantly following incubation with miRNA-206 inhibitor (Fig. 4E, $\mathrm{P}<0.01)$. This displayed the specific efficiency of miRNA-206 mimics and inhibitor; collectively, these data indicate that miRNA-206 can directly bind to lnc00152 through miRNA recognition sites.

Inc00152 regulates the miRNA-206 target gene, NRP1. Yin et al (28) previously demonstrated that miRNA-206

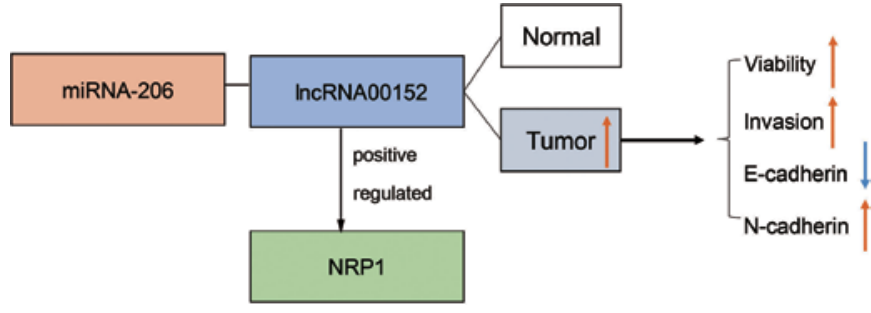

Figure 6. A schematic model demonstrating the mechanisms through which lnc00152 functions as a competing endogenous RNA (ceRNA) to regulate NRP1 expression by sponging miRNA-206 in colorectal cancer (CRC). lnc00152 was highly expressed in CRC tumor tissues compared with adjacent normal tissues and promoted CRC cell viability and invasion by competitively binding miRNA-206 upregulating NRP1, and subsequentlyupregulating $\mathrm{N}$-cadherin, while downregulating E-cadherin.

significantly inhibited the luciferase activity of the RLuc-NRP1 3'-UTR reporter, confirming that NRP1 is a target gene of miR-206. Thus, one of our aims in this study was to clarify whether lnc00152 regulates NRP1 in CRC cells. Our results indicated that $\operatorname{lnc} 00152$ silencing downregulated NRP1 expression, while lnc00152 overexpression elevated NRP1 expression (Fig. 5A and B, P<0.01). Additionally, we validated the effects of 1 nc00152 expression on EMT in CRC cells. Inc00152 silencing downregulated $\mathrm{N}$-cadherin expression, whereas it upregulated E-cadherin expression (Fig. 5A-D, $\mathrm{P}<0.01$ ). By contrast, Inc00152 overexpression increased $\mathrm{N}$-cadherin expression, while it decreased E-cadherin expression (Fig. 5A-D, $\mathrm{P}<0.01$ ). Therefore, lnc00152 regulates NRP1 expression and additionally affects EMT in CRC cells.

The mechanisms through which $\operatorname{lnc} 00152$ regulates NRP1 in colorectal cancer. From the above-mentioned results, we drew a schematic model to demonstrate the possible mechanisms through which lnc00152 regulates NRP1 (Fig. 6). Compared with the adjacent normal tissues, $\operatorname{lnc} 00152$ was highly expressed in CRC tumor tissues and promoted CRC cell viability and invasion by competitively binding miRNA-206, upregulating NRP1, and subsequently upregulating N-cadherin, while downregulating E-cadherin.

\section{Discussion}

In this study, we found that $\operatorname{lnc} 00152$ was expressed in colorectal tumor tissues and the CRC cell lines, SW480, LoVo and SW620. The regulation of $\operatorname{lnc} 00152$ also affected CRC cell viability, invasion and EMT in vitro, as well as tumor growth in vivo. This process was associated with NRP1, a miRNA-206 target gene, and miRNA-206 was associated with lnc00152 expression.

lncRNAs play critical roles in cancer development and progression (29). Recent studies have indicated that lncRNAs can regulate both the migration and invasiveness of CRC. For example, the downregulation of IncRNA H19 has been shown to inhibit CRC cell migration and metastasis (30). This is consistent with our findings in that the silencing of lnc00152 decreased the proliferative and invasive ability of CRC cells. IncRNAs have also been reported to suppress EMT in CRC (11). EMT is a hallmark in tumor progression (31). 
Our findings further validated this observation by showing that the downregulation of 1 nc00152 reduced $\mathrm{N}$-cadherin and increased E-cadherin expression, while lnc00152 overexpression had the opposite effect. This is consistent with the role of other lncRNAs in the CRC cell lines LoVo and HCT116 (11).

Furthermore, we found that overexpression of lnc00152 may increase the malignant potential of CRC cells. This has also been observed in a previous study which showed that the upregulation of IncRNA BANCR was associated with metastasis and the poor survival of patients with CRC (12). In this study, patients with a higher level of lnc00152 had a shorter survival time, displaying an association between the lnc00152 level and CRC prognosis. This finding, too, is supported by the findings of other studies that have demonstrated an unfavorable prognosis of patients with CRC with a lncRNA expression (32-34). In our tumor xenograft experiment, lnc00152 overexpression promoted tumor growth, which demonstrates that lnc00152 plays an essential role in regulating the malignancy of CRC. Importantly, as lnc00152 silencing appears to have an anti-tumor effect, it may function as a therapeutic target in the treatment of CRC, and may thus aid in the treatment and prognosis of CRC.

Some miRNAs have been observed to be dysregulated in CRC tissues (35). The function of miRNAs in CRC involves controlling EMT (36) and affecting cell proliferation, migration and invasion (37), similar to the function of lncRNAs in CRC. In ovarian cancer, the miRNA-lncRNA signature is important in patient survival (38). The miRNA-lncRNA complex is also involved in the transformation process of gastric cancer initiation to malignancy (39). Accordingly, lncRNA may interact with miRNAs to regulate colorectal tumor malignancy. Previous studies have reported that 1 nc00152 is implicated in CRC by regulating cell cycle, apoptosis, cell motility and EMT $(40,41)$. In addition, Inc00152 has been demonstrated to promote oncogenesis via several mechanisms, including serving as a sponge for miR-4767 and miR-205 (42,43) and a partner for EZH2 (41). In this study, we observed the interaction of lnc00152 with miRNA-206 with a dual luciferase assay. This observation and other reports of miR-206 inhibiting tumor invasion and migration in CRC (44), highlight an important association between miRNA-206 and lnc00152 in CRC.

Our experiments have additionally located the mutation site on lnc00152 that interacts with miRNA-206. NRP1 is the target gene of miRNA-206 (28), and our data demonstrated that regulating the 1 co0 0152 levels altered NRP1 expression. NRP1 is a co-receptor for vascular endothelial growth factor (VEGF), and the blockaded of NRP1 suppresses tumor growth by inhibiting angiogenesis or by directly inhibiting tumor cell proliferation (45). In CRC, NRP1 is associated with liver metastasis (46), and, importantly, EMT in CRC is dependent on NRP1 (26). Future studies, therefore, are warranted in order to focus on the molecular mechanisms that underlie the synergic effects of miRNA-206 and lnc00152 on CRC progression and on the signaling pathway of the NRP1-associated modulation of CRC metastasis.

In conclusion, the results of the present study identify lnc00152 as a competing endogenous RNA that positively regulates NRP1 expression by sponging miRNA-206 (Fig. 6). Given its critical role in $\mathrm{CRC}$, it may be an effective therapeutic target in CRC prognosis and treatment.

\section{Acknowledgements}

Not applicable.

\section{Funding}

No funding was received.

\section{Availability of data and materials}

All data generated or analyzed during this study are included in this published article.

\section{Authors' contributions}

ZPC, JCW and QW conceived and designed the research and drafted the manuscript. PY, WLL and JBZ conducted the experiments. $\mathrm{FH}, \mathrm{HCC}$ and $\mathrm{HH}$ performed the data analysis. JC substantially contributed to the design and conception of the study, and critically reviewed and revised the manuscript, and approved the final version to be published. All authors have read and approved the final manuscript.

\section{Ethics approval and consent to participate}

The use of human samples was approved by the Ethics Committee Guangzhou First People's Hospital, Guangzhou, China and all patients involved in the study provided written informed consent. The use of animals in this study was approved by the Ethics Committee of the Laboratory Animal Center of South China University of Technology, Guangzhou, China.

\section{Consent for publication}

Not applicable.

\section{Competing interests}

The authors declare that they have no competing interests.

\section{References}

1. Siegel R, Desantis C and Jemal A: Colorectal cancer statistics, 2014. CA Cancer J Clin 64: 104-117, 2014

2. Kanthan R, Senger JL and Kanthan SC: Molecular events in primary and metastatic colorectal carcinoma: A review. Pathol Res Int 2012: 597497, 2012.

3. Guan X, Jiang Z, Ma T, Liu Z, Hu H, Zhao Z, Song D, Chen Y, Wang $G$ and Wang $X$ : Radiotherapy dose led to a substantial prolongation of survival in patients with locally advanced rectosigmoid junction cancer: A large population based study. Oncotarget 7: 28408-28419, 2016.

4. Zhu DJ, Chen XW, OuYang MZ and Lu Y: Three surgical planes identified in laparoscopic complete mesocolic excision for right-sided colon cancer. World J Surg Oncol 14: 7, 2016.

5. Wang YW, Huang LY, Song CL, Zhuo CH, Shi DB, Cai GX, $\mathrm{Xu}$ Y, Cai SJ and Li XX: Laparoscopic vs open abdominoperineal resection in the multimodality management of low rectal cancers. World J Gastroenterol 21: 10174-10183, 2015.

6. Lin T, Song C, Chuo DY, Zhang H and Zhao J: Clinical effects of autologous dendritic cells combined with cytokine-induced killer cells followed by chemotherapy in treating patients with advanced colorectal cancer: A prospective study. Tumour Biol 37: 4367-4372, 2016. 
7. Du XH, Liu HL, Li L, Xia SY, Ning N, Zou ZY, Teng D, Xiao CH, $\mathrm{Li}$ R and Xu YX: Clinical significance of immunotherapy with combined three kinds of cells for operable colorectal cancer. Tumour Biol 36: 5679-5685, 2015.

8. Chen LL and Zhao JC: Functional analysis of long noncoding RNAs in development and disease. Adv Exp Med Biol 825: 129-158, 2014.

9. Hu W, Yuan B, Flygare J and Lodish HF: Long noncoding RNA-mediated anti-apoptotic activity in murine erythroid terminal differentiation. Genes Dev 25: 2573-2578, 2011.

10. Rossi MN and Antonangeli F: LncRNAs: New players in apoptosis control. Int J Cell Biol 2014: 473857, 2014.

11. Zhang M, Wu WB, Wang ZW and Wang XH: lncRNA NEAT1 is closely related with progression of breast cancer via promoting proliferation and EMT. Eur Rev Med Pharmacol Sci 21: 1020-1026, 2017.

12. Shen X, Bai Y, Luo B and Zhou X: Upregulation of lncRNA BANCR associated with the lymph node metastasis and poor prognosis in colorectal cancer. Biol Res 50: 32, 2017.

13. Zhou HB, Li Q, Liu M, Cao YQ and Xu JY: Increased expression of long non-coding RNA SBDSP1 correlates with poor survival in colorectal cancer. Eur Rev Med Pharmacol Sci 21: 3837-3841, 2017.

14. Chen DL, Chen LZ, Lu YX, Zhang DS, Zeng ZL, Pan ZZ, Huang $\mathrm{P}$, Wang $\mathrm{FH}$, Li YH, Ju HQ, et al: Long noncoding RNA XIST expedites metastasis and modulates epithelialmesenchymal transition in colorectal cancer. Cell Death Dis 8: e3011, 2017.

15. Wu K, Zhao Z, Ma J, Chen J, Peng J, Yang S and He Y: Deregulation of miR-193b affects the growth of colon cancer cells via transforming growth factor- $\beta$ and regulation of the SMAD3 pathway. Oncol Lett 13: 2557-2562, 2017.

16. Tong F, Ying Y, Pan H, Zhao W, Li H and Zhan X: MicroRNA-466 (miR-466) functions as a tumor suppressor and prognostic factor in colorectal cancer (CRC). Bosn J Basic Med Sci: Jan 17, 2018 (Epub ahead of print). doi: 10.17305/bjbms.2018.2376.

17. Hsiao KY, Lin YC, Gupta SK, Chang N, Yen L, Sun HS and Tsai SJ: Noncoding effects of circular RNA CCDC66 promote colon cancer growth and metastasis. Cancer Res 77: 2339-2350, 2017.

18. Ebrahimi F, Gopalan V, Wahab R, Lu CT, Smith RA and Lam AK: Deregulation of miR-126 expression in colorectal cancer pathogenesis and its clinical significance. Exp Cell Res 339: 333-341, 2015.

19. Li DY, Chen WJ, Luo L, Wang YK, Shang J, Zhang Y, Chen G and Li SK: Prospective lncRNA-miRNA-mRNA regulatory network of long non-coding RNA LINC00968 in non-small cel lung cancer A549 cells: A miRNA microarray and bioinformatics investigation. Int J Mol Med 40: 1895-1906, 2017.

20. Guo PD, Lu XX, Gan WJ, Li XM, He XS, Zhang S, Ji QH, Zhou F, Cao Y, Wang JR, et al: RAR $\gamma$ downregulation contributes to colorectal tumorigenesis and metastasis by derepressing the Hippo-Yap pathway. Cancer Res 76: 3813-3825, 2016.

21. Wang JR, Gan WJ, Li XM, Zhao YY, Li Y, Lu XX, Li JM and Wu H: Orphan nuclear receptor Nur77 promotes colorecta cancer invasion and metastasis by regulating MMP-9 and E-cadherin. Carcinogenesis 35: 2474-2484, 2014.

22. Ye GD, Sun GB, Jiao P, Chen C, Liu QF, Huang XL, Zhang R, Cai WY, Li SN, Wu JF, et al: OVOL2, an inhibitor of WNT signaling, reduces invasive activities of human and mouse cancer cells and is down-regulated in human colorectal tumors. Gastroenterology 150: 659-671.e16, 2016.

23. Waszkiewicz N, Szajda SD, Konarzewska-Duchnowska E, Zalewska-Szajda B, Gałązkowski R, Sawko A, Nammous H, Buko V, Szulc A, Zwierz K, et al: Serum $\beta$-glucuronidase as a potential colon cancer marker: A preliminary study. Postepy Hig Med Dosw 69: 436-439, 2015.

24. Miyauchi JT, Chen D, Choi M, Nissen JC, Shroyer KR, Djordevic S, Zachary IC, Selwood D and Tsirka SE: Ablation of Neuropilin 1 from glioma-associated microglia and macrophages slows tumor progression. Oncotarget 7: 9801-9814, 2016.

25. Li X, Fan S, Pan X, Xiaokaiti Y, Duan J, Shi Y, Pan Y, Tie L, Wang X, Li Y, et al: Nordihydroguaiaretic acid impairs prostate cancer cell migration and tumor metastasis by suppressing neuropilin 1. Oncotarget 7: 86225-86238, 2016.

26. Tomida C, Yamagishi N, Nagano H, Uchida T, Ohno A, Hirasaka K, Nikawa T and Teshima-Kondo S: Antiangiogenic agent sunitinib induces epithelial to mesenchymal transition and accelerates motility of colorectal cancer cells. J Med Invest 64: 250-254, 2017
27. Livak KJ and Schmittgen TD: Analysis of relative gene expression data using real-time quantitative PCR and the 2(-Delta Delta C(T)) Method. Methods 25: 402-408, 2001.

28. Yin K, Yin W, Wang Y, Zhou L, Liu Y, Yang G, Wang J and Lu J: MiR-206 suppresses epithelial mesenchymal transition by targeting TGF- $\beta$ signaling in estrogen receptor positive breast cancer cells. Oncotarget 7: 24537-24548, 2016.

29. Prensner JR and Chinnaiyan AM: The emergence of lncRNAs in cancer biology. Cancer Discov 1: 391-407, 2011.

30. Liang W, Zou Y, Qin F, Chen J, Xu J, Huang S, Chen J and Dai S: sTLR4/MD-2 complex inhibits colorectal cancer migration and invasiveness in vitro and in vivo by lncRNA H19 downregulation. Acta Biochim Biophys Sin (Shanghai) 49: 1035-1041, 2017.

31. Cao H, Xu E, Liu H, Wan L and Lai M: Epithelial-mesenchymal transition in colorectal cancer metastasis: A system review. Pathol Res Pract 211: 557-569, 2015.

32. Chen SW, Zhu J, Ma J, Zhang JL, Zuo S, Chen GW, Wang X, Pan YS, Liu YC and Wang PY: Overexpression of long non-coding RNA H19 is associated with unfavorable prognosis in patients with colorectal cancer and increased proliferation and migration in colon cancer cells. Oncol Lett 14: 2446-2452, 2017.

33. Gao X, Wen J, Gao P, Zhang G and Zhang G: Overexpression of the long non-coding RNA, linc-UBC1, is associated with poor prognosis and facilitates cell proliferation, migration, and invasion in colorectal cancer. OncoTargets Ther 10: 1017-1026, 2017.

34. Lu M, Liu Z, Li B, Wang G, Li D and Zhu Y: The high expression of long non-coding RNA PANDAR indicates a poor prognosis for colorectal cancer and promotes metastasis by EMT pathway. J Cancer Res Clin Oncol 143: 71-81, 2017.

35. Chen X, Shi K, Wang Y, Song M, Zhou W, Tu H and Lin Z: Clinical value of integrated-signature miRNAs in colorectal cancer: miRNA expression profiling analysis and experimental validation. Oncotarget 6: 37544-37556, 2015.

36. Jaca A, Govender P, Locketz M and Naidoo R: The role of miRNA-21 and epithelial mesenchymal transition (EMT) process in colorectal cancer. J Clin Pathol 70: 331-356, 2017.

37. Deng B, Wang B, Fang J, Zhu X, Cao Z, Lin Q, Zhou L and Sun X: MiRNA-203 suppresses cell proliferation, migration and invasion in colorectal cancer via targeting of EIF5A2. Sci Rep 6: 28301, 2016.

38. Guo L, Peng Y, Meng Y, Liu Y, Yang S, Jin H and Li Q: Expression profiles analysis reveals an integrated miRNA-lncRNA signature to predict survival in ovarian cancer patients with wild-type BRCA1/2. Oncotarget 8: 68483-68492, 2017.

39. Mao Y, Liu R, Zhou H, Yin S, Zhao Q, Ding X and Wang H: Transcriptome analysis of miRNA-lncRNA-mRNA interactions in the malignant transformation process of gastric cancer initiation. Cancer Gene Ther 24: 267-275, 2017.

40. Zhao J, Liu Y, Zhang W, Zhou Z, Wu J, Cui P, Zhang Y and Huang G: Long non-coding RNA Linc00152 is involved in cell cycle arrest, apoptosis, epithelial to mesenchymal transition, cell migration and invasion in gastric cancer. Cell Cycle 14: 3112-3123, 2015.

41. Chen WM, Huang MD, Sun DP, Kong R, Xu TP, Xia R, Zhang EB and Shu YQ: Long intergenic non-coding RNA 00152 promotes tumor cell cycle progression by binding to EZH2 and repressing p15 and p21 in gastric cancer. Oncotarget 7: 9773-9787, 2016

42. Wang Y, Liu J, Bai H, Dang Y, Lv P and Wu S: Long intergenic non-coding RNA 00152 promotes renal cell carcinoma progression by epigenetically suppressing P16 and negatively regulates miR-205. Am J Cancer Res 7: 312-322, 2017.

43. Teng W, Qiu C, He Z, Wang G, Xue Y and Hui X: Linc00152 suppresses apoptosis and promotes migration by sponging miR-4767 in vascular endothelial cells. Oncotarget 8: 85014-85023, 2017.

44. Sun P, Sun D, Wang X, Liu T, Ma Z and Duan L: miR-206 is an independent prognostic factor and inhibits tumor invasion and migration in colorectal cancer. Cancer Biomark 15: 391-396, 2015.

45. Jubb AM, Strickland LA, Liu SD, Mak J, Schmidt M and Koeppen H: Neuropilin-1 expression in cancer and development. J Pathol 226: 50-60, 2012.

46. Zhang Y, He X, Liu Y, Ye Y, Zhang H, He P, Zhang Q, Dong L, Liu Y and Dong J: microRNA-320a inhibits tumor invasion by targeting neuropilin 1 and is associated with liver metastasis in colorectal cancer. Oncol Rep 27: 685-694, 2012. 